\title{
Efficient Protection of Response Messages in DTLS-Based Secure Multicast Communication
}

\author{
Marco Tiloca \\ SICS Swedish ICT AB, Security Lab \\ Isafjordsgatan 22, Kista, Sweden \\ Phone: +460706046501 Fax: +46897517230 \\ marco@sics.se
}

\begin{abstract}
DTLS is a standardized security protocol designed to provide endto-end secure communication among two peers, and particularly considered for the emerging Internet of Things. In order to protect group communication, the IETF is currently working on a method to secure multicast messages through the same DTLS security services. However, such an approach relies on traditional DTLS sessions to protect unicast responses to multicast messages. This increases the amount of security material stored by group members and can have a relevant impact on network performance. In this paper we propose an extension to the IETF approach which allows to efficiently protect group responses by reusing the same group key material. Our proposal does not require to establish additional DTLS sessions, thus preserving high communication performance within the group and limiting storage overhead on group members. Furthermore, we discuss a suitable key management policy to provision and renew group key material.
\end{abstract}

\section{Categories and Subject Descriptors}

C.2.0 [Computer-Communication Networks]: General-Security and protection

\section{General Terms SECURITY}

\section{Keywords}

Security, DTLS, Multicast, Group communication

\section{INTRODUCTION}

The Datagram Transport Layer Security (DTLS) protocol [7] has been introduced by the IETF to provide secure communication between two peers, in the presence of unreliable datagram protocols such as UDP [10]. Although it has been standardized only recently,

This work was carried out during the tenure of an ERCIM "Alain Bensoussan" Fellowship Programme. The research leading to these results has received funding from the European Union Seventh Framework Programme (FP7/2007-2013) under grant agreement $\mathrm{n}^{\circ} 246016$
DTLS is already asserting itself as the de-facto security protocol to protect end-to-end communication in the emerging Internet of Things (IoT) [14][15][20]. DTLS has been designed to be as much similar as possible to the widely adopted $T L S$ protocol [18], and provides equivalent security services, i.e. it allows client and server applications to communicate with one another preventing eavesdropping, tampering, and message forgery. In order to establish a DTLS session, two peers perform a preliminary message exchange known as handshake, so agreeing on a ciphersuite and establishing common security material.

More recently, the lighthweight Constrained Application Protocol (CoAP) [22] developed by the IETF working group CoRE [1] has specifically mandated the adoption of DTLS to provide secure communication in machine-to-machine applications and constrained networks. Furthermore, the CoRE working group has also considered application scenarios relying on group communication, and is currently defining how to use the CoAP protocol on top of IP multicast [4]. This is particularly desirable and convenient in the presence of Low-Power and Lossy Networks (LLNs), which are typically composed of low-power and resource constrained devices featuring scarce computing power and limited battery life.

Of course, such application scenarios may also require the presence of reliable and efficient security services, in order to assure secure multicast communication within a group. This is the reason why another IETF working group, namely DICE [2], is currently defining how to adapt the DTLS protocol to protect multicast messages sent by a sender node and received by multiple listener nodes in the same group [17]. Such an approach has the main goal of protecting multicast group communication through the usual DTLS security services. In addition, it relies on a single set of group key material shared among all group members, and does not require to perform the complex and costly DTLS handshake in order to establish a multicast secure session.

The approach described in [17] considers also the protection of group responses sent by listener nodes as unicast replies to multicast messages. That is, group responses are supposed to be protected through distinct unicast DTLS sessions, established between sender nodes and replying listener nodes in the group. This may require to perform a significant number of DTLS handshakes, and may be not reasonable in case unicast secure communication is required only to protect group responses. Also, such an approach is likely to be inefficient and even not feasible, especially in the presence of low-power constrained devices, due to the complex DTLS handshake execution, and the resulting computing and communication overhead. Finally, group members would be required to store a 
considerable amount of supplementary security material in order to maintain additional, possibly unnecessary, unicast DTLS sessions.

In this paper, we propose an extension to the DTLS-based multicast communication method under development by the IETF, and describe how to efficiently protect group responses according to the same group communication scheme and by reusing the same group key material. By doing so, it is not necessary to establish any unicast DTLS session, hence avoiding to perform additional and costly DTLS handshakes, and preserving high communication performance within the group. In addition, our extension does not require group members to store and maintain any additional security material, thus not affecting them in terms of storage overhead.

To the best of our knowledge, this paper represents the first contribution aimed at improving efficiency of group response protection in DTLS-based group communication. Also, our approach does not break current standards, and thus is suitable for a possible integration in the current multicast scheme proposed by the IETF. As a further contribution, the paper describes a suitable key management policy to address provisioning and renewal of group key material, in the presence of DTLS-based multicast communication.

The rest of the paper is organized as follows. Section 2 briefly overviews the main features of DTLS, while Section 3 describes the DTLS-based multicast communication method proposed by the IETF. In Section 4, we present our extension for reusing group key material to protect group responses. Section 5 discusses a key management policy suitable to the considered communication scenario. Finally, in Section 6 we draw our conclusive remarks.

\section{DTLS OVERVIEW}

The Datagram Transport Layer Security (DTLS) protocol [7] has been designed to provide secure communication for applications based on unreliable datagram protocols, such as UDP [10]. DTLS is based on the $T L S$ protocol [18], and provides equivalent security services, i.e. it allows client and server applications to communicate with one another preventing eavesdropping, tampering, and message forgery. In order to address the unreliable nature of datagram transport protocols, DTLS introduces a few minor changes with respect to TLS, such as making distinct messages independent from one another, and not forcing to terminate active sessions upon receiving invalid messages. Also, DTLS provides an optional stateless Cookie mechanism, in order to counteract possible Denial of Service $(D o S)$ attacks against DTLS servers.

\begin{tabular}{|c|c|c|c|c|}
\hline Type & Version & Epoch & $\begin{array}{c}\text { Sequence } \\
\text { Number }\end{array}$ & Length \\
\hline 1 Byte & 2 Bytes & 2 Bytes & 6 Bytes & 2 Bytes
\end{tabular}

Figure 1: DTLS record header.

Messages are transmitted as a sequence of DTLS records, each one of which includes the header shown in Figure 1. The Type and Version fields indicate the referred higher level protocol and the adopted version of DTLS, respectively, while the Length field represents the size in bytes of the actual application data conveyed in the record. Finally, with respect to TLS, two additional fields have been included, namely Epoch and Sequence Number. Specifically, the Epoch value is incremented upon changing the currently used security protocols and material, while the Sequence Number value is incremented for every new message transmitted by the same peer over the same DTLS session. The concatenation of the Epoch and Sequence Number fields is considered as a 64 bit fresh value, namely explicit nonce, and is used to compute a Message Authentication Code (MAC) to provide integrity of DTLS records.

For each secure session established, a DTLS peer maintains a write connection state and a read connection state. Practically, write (read) connection states are operating environments referred while processing outgoing (incoming) messages within a DTLS session. Among other information, connection states define a given DTLS peer as either client or server, and include current Epoch and Sequence Number values, as well as algorithms and security material used throughout the session.

A DTLS client and server establish a secure session through a specific handshake process. This is necessary in order to agree on a common set of security algorithms and derive the security material used to protect DTLS records. In particular, DTLS client and server generate and exchange two random values, i.e. client random and server random, and come to agree on a premaster secret. The latter is in turn composed of a 46 bytes random field, whose value is generated at random, and a 2 bytes client version field. Then, the premaster secret is used together with client random and server random by the two peers, in order to derive the actual security material for the DTLS session.

However, performing a DTLS handhshake is a complex, time consuming, and resource demanding process. In fact, it results in at least two message round trips, requires to perform a number of cryptographic operations, and assumes that DTLS client and server have been already provided with some preliminary security material. In particular, two main approaches to provide preinstalled cryptographic keys are admitted. The first one is based on asymmetric key pairs, while the second one relies on symmetric keys pre-shared between client and server and avoids sending/receiving public certificates and performing costly public key operations.

\section{DTLS-BASED MULTICAST SECURITY}

The IETF is currently working on readapting DTLS to secure group communication, with particular reference to Low-Power and Lossy Networks (LLNs) and the lightweight application protocol CoAP [4][22]. Specifically, the IETF working group DICE [2] is defining how to adapt the usual DTLS record security services in order to protect multicast messages sent by a sender node and received by multiple listener nodes in the same group [17]. This section summarizes the main aspects of such a proposal.

In the following, we consider a multicast group $G$ associated to a unique IP address $I P_{G}$, which has been previously allocated for IP multicast communication. All group members trust one another, and are not supposed to tamper with multicast messages sent within the group. In particular, members of group $G$ can belong to two possible categories, namely senders and listeners, and can have both roles depending on their actual network activity in the group. Sender nodes are responsible for transmitting multicast messages to group $G$, while listener nodes are explicitly interested in receiving multicast messages. In principle, any node can become a listener, by registering with a network routing device, and signaling its intent to receive messages sent to the $I P_{G}$ address. Instead, senders nodes are not supposed to be aware of the group membership or get notified of new listener nodes.

Also, members of group $G$ maintain separate group connection 
states $C S^{W}$ and $C S^{R}$, referred while processing outgoing or incoming multicast messages within the group, respectively. In particular, group connection states are configured in such a way that sender nodes act as servers, while listener nodes act as clients. Every sender node in the group initializes to 0 the Sequence Number value in its own write group connection state $C S^{W}$, and increments it for every multicast DTLS record sent to group $G$.

Finally, an additional entity named Group Controller $(G C)$ is responsible for creating the group, managing the actual join process to add new group members, and providing them with the commonly shared group security material. The $G C$ is not required to be also an actual sender within the group, and can be discovered by joining nodes through various methods, such as DNS-SD [16] and Resource Directory [21].

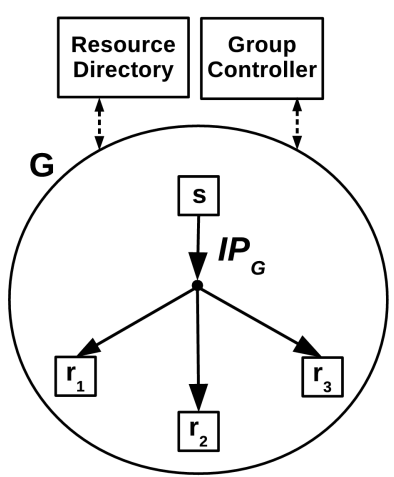

(a)

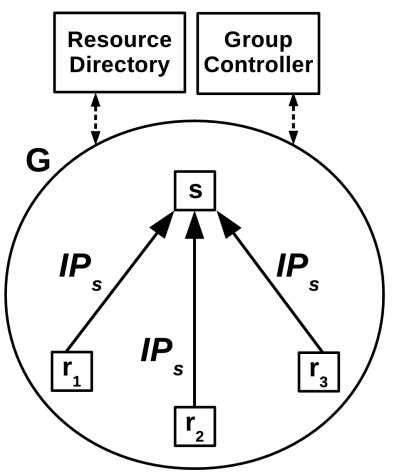

(b)
Figure 2: Group communication scenario: (a) single multicast message; (b) multiple unicast responses.

Figure 2 shows a group $G$ composed of one sender node $s$ and three listener nodes $r_{1}, r_{2}$, and $r_{3}$. Group messages are sent by the sender node $s$ as a single multicast transmission (Figure 2:a), while possible responses by individual listener nodes are sent back as unicast messages (Figure 2:b). This is consistent with the CoAP group communication guidelines provided in [4].

\subsection{Protection of multicast messages}

As described in [17], a DTLS group session is established without performing a regular handshake process. Instead, the $G C$ securely provides all group members with a common Group Security Association (GSA) [19], including the adopted ciphersuites and a set of preliminary security parameters. Specifically, all group members are provided by the $G C$ with the same GSA, and use it to autonomously derive the same DTLS SecurityParameters structure as defined in [18]. Besides, the GSA includes also the DTLS premaster secret, the client random value and the server random value, usually generated while performing a DTLS handshake. Then, every group member relies on such three pieces of information in order to derive the actual group key material reported below. Note that, as an alternative, the $G C$ may include the six key material elements directly in the GSA, upon providing it to group members.

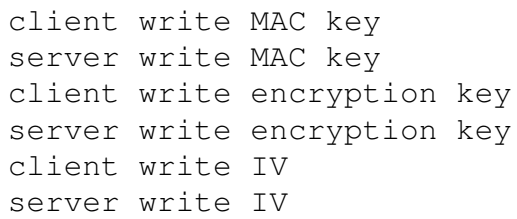

Such group key material is thus associated to the GSA, and secretly shared between all group members. Then, sender nodes can use it to protect multicast messages by means of usual DTLS security services. Specifically, before transmitting a multicast message $M$, a sender node protects it by using the group server write parameters. Then, every listener node uses the same server write parameters to process message $M$ upon its reception. The exact usage of the server write parameters depends on the specific ciphersuite adopted by group members [17]. On the other hand, at the moment it is not defined any possible usage of the client write parameters, either by sender nodes, or by listener nodes. Finally, note that, since all group members share the same group security material, it is not possible to assure source authenticity of multicast messages sent within the group.

As mentioned in Section 2, the message authenticity process relies on a 64 bit explicit nonce, i.e the concatenation of the Epoch field and the Sequence Number field in the DTLS record header. However, the presence of multiple sender nodes inevitably leads to reusing the same explicit nonce values, and makes it impossible to check that received multicast messages are actually fresh. As a consequence, multicast messages sent by different sender nodes may be considered as replayed messages by listener nodes, and get discarded. Furthermore, the commonly adopted ciphersuite MTS_WITH_AES_128_CCM_8 requires that the explicit nonce value is different for each distinct invocation of the encryption function using the same key material. Hence, DTLS sequence number values maintained by multiple sender nodes in their respective write group connection state $C S^{W}$ would need to be synchronized, in order to avoid their reuse.

Since synchronizing sequence number spaces within a group can definitely be a difficult task, [17] proposes to separate sequence number spaces of different sender nodes, and then embed a unique sender identifier in the original Sequence Number field of the DTLS record header, as suggested in [11]. Then, the $G C$ is required to additionally assign a unique SenderID to each sender node in the group, and provide all group members with a list of active SenderIDs. Also, every listener node maintains a distinct read group connection state $C S_{s}^{R}$ for every sender node $s$, identified by the associated SenderID. Finally, the original DTLS record header is adapted accordingly, as reported in Figure 3. In particular, the original 6 octet Sequence Number field in the DTLS record header has been redefined as a 1 octet SenderID field followed by a 5 octet Truncated Sequence Number field.

\begin{tabular}{|c|c|c|c|c|c|}
\hline Type & Version & Epoch & SenderID & $\begin{array}{c}\text { Truncated } \\
\text { Sequence Number }\end{array}$ & Length \\
\hline 1 Byte & 2 Bytes & 2 Bytes & 1 Byte & 5 Bytes & 2 Bytes
\end{tabular}

Figure 3: DTLS record header for multicast messages.

Upon receiving a multicast message $M$, every listener node retrieves i) the destination address $I P_{G}$ as well as the destination port $P_{G}$; and ii) the sender identifier from the SenderID field of the DTLS record header. Then, it uses such pieces of information to retrieve the GSA associated to group $G$ and the read group connection state $C S_{s}^{R}$ referred to the sender node $s$. Finally, it retrieves the group server write parameters, and uses them to process message $M$. The message freshness is verified by checking the Epoch and Truncated Sequence Number field with the values stored in the read group connection state $C S_{s}^{R}$. 
Note that listener nodes which lately join the group do not know the Epoch and Truncated Sequence Number currently used by different sender nodes. Hence, when such listener joining nodes receive a multicast message, they may be not able to verify if it is fresh and has not been replayed by an adversary. In order to overcome this issue, [17] suggests to adopt the following approach. Upon receiving a multicast message $M$ from a given sender node $s$ for the first time, a late joining listener node initializes the Epoch and Truncated Sequence Number in its own read group connection state $C S_{s}^{R}$ associated to the sender node $s$. However, message $M$ is discarded, i.e. it is not delivered to the application layer. This provides a reference point to identify if future multicast messages from the same sender node $s$ are fresher than the last one received.

\section{GROUP RESPONSE PROTECTION}

As summarized in Section 3, the approach described in [17] extensively addresses the protection of multicast messages sent by sender nodes. However, in many application scenarios, listener nodes are supposed to reply to sender nodes, by sending back individual response messages. Besides, the latters may need to be secured as well, in order to assure confidentiality, integrity, authenticity, and replay protection. In fact, if such responses to multicast messages were not secured at all, an adversary could access their content as well as tamper with them by forging data and control information, so performing an attack against the whole group.

The current version of [17] takes this issue into account and prescribes that unicast responses to DTLS-based multicast messages must be secured. In particular, such unicast responses are supposed to be protected by means of traditional unicast DTLS sessions, established between sender nodes and listener nodes. However, this may require to perform a significant number of DTLS handshakes within the group, and may be not convenient in case unicast secure communication is required only to protect group responses. In this section, we present an extension to the secure communication scheme described in [17], and propose an approach to efficiently protect group responses, by relying on the same scheme described in Section 3 and reusing the same group security material.

\subsection{Motivation}

The straightforward approach described in [17] and based on traditional unicast DTLS sessions requires that every sender node in the group establishes a unicast DTLS session with each replying listener node. Hence, every sender node is required to perform a DTLS handshake with all replying listener nodes in the group, i.e., in the worst case, with all other group members.

However, this can be not reasonable and convenient, especially if unicast secure communication within the group is required only by listener nodes to send group responses to multicast messages. Moreover, such an approach is likely to be inefficient and even not feasible, especially in the presence of constrained low-power devices, due to the complexity displayed by the DTLS handshake execution, and the resulting computing and communication overhead.

In fact, as a first option, every sender node should establish a unicast DTLS session with each replying listener node before sending its first multicast message to the group. This would require every sender node to be aware of the current group membership, i.e. to know what listener nodes are currently present in the group. Also, every sender node should establish further unicast DTLS sessions in case new listener nodes join the group. Hence, this would force to provide sender nodes with otherwise unnecessary information, as well as to keep them up to date about possible changes in the group membership, upon new nodes' joining. However, [17] explicitly states that applications on group nodes do not know, and do not get notified, when new listener nodes join the group.

As a different approach, every listener node should establish a unicast DTLS session with a given sender node in the group before sending back its own first group response message. However, this would result in sender nodes possibly flooded with a potentially consistent number of DTLS handshakes to be performed.

Note that both the approaches described above also introduce a not negligible delay before the group can become fully operative, due to the execution of a considerable number of DTLS handshakes. Moreover, the observed impact on group members' availability and performance would be even more severe, in case nodes are allowed to be members of more than one multicast group at the same time.

\subsection{Protection of response messages}

In the following, we describe how listener nodes can protect their individual group responses, by reusing the same group security material described in Section 3.1.

Basically, in order to adopt our approach, sender nodes must be able to recognize whether a received unicast message is actually a group response sent by a listener node, i.e. a reply to a previously sent multicast message. Hence, upon creating the group $G$, the $G C$ provides group nodes also with a unique 1 byte GroupID associated to $G$, namely $I D_{G}$. After that, possible new nodes must be provided with the same identifier $I D_{G}$ upon joining the group. So doing, every group member is able to univocally identify the GSA associated to $G$, by means of the associated multicast address $I P_{G}$ and port $P_{G}$, as well as by the GroupID ID $D_{G}$.

In addition, every sender node maintains a separate read group connection state $C S_{r}^{R}$ identified by the unicast address $I P_{r}$, for every listener node $r$ that has sent at least one group response. Similarly, every listener node maintains a separate write group connection state $C S_{s}^{W}$, for every sender node $s$ in the group, identified by the unique associated SenderID $I D_{s}$. Each one of such $C S_{s}^{W}$ includes a Sequence Number value initialized to 0 and incremented for every unicast group response sent to sender node $s$. Finally, we introduce an additional format for the DTLS record header, whose structure is depicted in Figure 4.

\begin{tabular}{|c|c|c|c|c|c|}
\hline Type & Version & Epoch & GrouplD & $\begin{array}{c}\text { Truncated } \\
\text { Sequence Number }\end{array}$ & Length \\
\hline 1 Byte & 2 Bytes & 2 Bytes & 1 Byte & 5 Bytes & 2 Bytes
\end{tabular}

Figure 4: DTLS record header for group response messages.

Listener nodes. Upon receiving a multicast message $M$ sent to the multicast address $I P_{G}$ by a sender node $s$, a listener node $r$ can process its group response $R$ as follows. First, it prepares a DTLS record specifying the actual response data as payload, and referring to the record header structure depicted in Figure 4. In particular, the listener node $r$ : i) fills the 5 octet Truncated Sequence Number field with the sequence number value stored in the write group connection state $C S_{s}^{W}$ associated to sender node $s$, before incrementing it; and ii) fills the 1 octet GroupID field with the group identifier $I D_{G}$. Finally, the listener node $r$ protects the group response $R$ by means of the group client write parameters, before sending it to the sender node $s$ as a unicast message. Note that, in case a 
sender node $s$ and a listener node $r$ have previously established a traditional unicast DTLS session, the latter can still be used to send back the response message $R$ in a secure way. Hence, in such a case, the listener node $r$ is not required to maintain a write group connection state $C S_{s}^{W}$ associated to the sender node $s$.

Sender nodes. Upon receiving the group response $R$, the sender node $s$ proceeds as follows. Firstly, it checks if any unicast DTLS session has been previously established with the listener node $r$, which is identified by the unicast source address $I P_{r}$ retrieved from message $R$. In case of positive match, the response message $R$ is processed according to the traditional DTLS protocol [7]. Note that, in such a case, the sender node $s$ does not need to maintain a read group connection state $C S_{r}^{R}$ associated to the listener node $r$. Conversely, in case of negative match, the sender node $s$ does not yet consider message $R$ to be invalid, and proceeds as follows.

First, the sender node $s$ parses the DTLS record header of message $R$ according to the format shown in Figure 4, and retrieves the value reported in the GroupID field, i.e. $I D_{G}$. Then, $s$ verifies whether it maintains a $G S A$ associated to the retrieved $I D_{G}$, namely $G S A_{G}$. In case of negative match, the sender node $s$ discards message $R$. Otherwise, it processes message $R$ by means of the group client write parameters associated to $G S A_{G}$. Then, it verifies the freshness of message $R$, by comparing the Epoch and Truncated Sequence Number field with the values stored in the read group communication state $C S_{r}^{R}$ associated to the listener node $r$. In case the read group communication state $C S_{r}^{R}$ cannot be found, the sender node $s$ creates it, and initializes Epoch and Truncated Sequence Number to the same values retrieved from message $R$. Note that, in case the response message $R$ has been received by a group member which is not configured as a sender node, the latter can simply consider $R$ to be invalid, and discard it.

In principle, it is possible that a sender node belongs to two or more different multicast groups whose respective $G C$ has assigned the same GroupID value. However, the sender node is supposed to communicate within each one of such different groups by means of distinct applications. In turn, each one of them would refer to a different port number to handle incoming messages. Hence, upon receiving a protected group response $R$, a sender node can still univocally identify the right $G S A$, by using the GroupI $D$ value retrieved from the DTLS header, together with the port number conveyed in the transport protocol header.

Finally, in the presence of our approach, the method proposed to deal with late listener joiners described in Section 3 should be used also for late sender joiners. In particular, upon receiving a group response $R$ from a given listener node $r$ for the first time, a late joining sender node creates and initializes a read group communication state $C S_{r}^{R}$ as described above. However, message $R$ is discarded, i.e. it is not delivered to the application layer. This provides a reference point to identify if future unicast group responses from the same listener node $r$ are fresher than the last one received.

\subsection{Discussion}

The main advantage of our approach consists in reusing the same group security material shared by all group members. This makes it possible for listener nodes in the group to securely reply to multicast messages without establishing new DTLS sessions. As a consequence, group members are not required to store additional security material, thus considerably limiting their storage overhead.
More important, unless unicast secure sessions are specifically required, group members do not need to perform any DTLS handshake. This particularly benefits sender nodes, which are not required to establish and maintain a unicast DTLS session with each replying listener node in the group. As a consequence, the group can become fully operative with no particular delays, and new joining nodes can instantly participate in multicast group communication. In addition, a considerable processing overhead is avoided on group members, and high communication performance is preserved within the group. To the best of our knowledge, there are currently no publicly available implementations of DTLS adaptations for multicast scenarios. As a future work, we will implement multicast communication support for DTLS, and experimentally evaluate performance in the presence and in the absence of the extension described in Section 4.2.

Furthermore, our approach requires that only a 1 byte group identifier is additionally provided to group members, upon joining the group. Also, it does not require sender nodes to be aware of the current group membership, i.e. to know what listener nodes are currently present in the group. Therefore, in dynamic application scenarios, it is not necessary to provide group members with the identity of new listener nodes when they join the group. Finally, the DTLS record header we propose for group responses (see Figure 4) is only 13 bytes in size, and has the very same structure of the DTLS record header proposed for multicast messages (see Figure 3). Hence, our approach is particularly easy to be integrated with the secure multicast scheme proposed by the IETF [17].

On the other hand, just like the approach presented in [17], our proposal does not provide data source authentication, since group responses to multicast messages are protected by means of the commonly shared group security material. Also, since all group members are potentially able to access and alter such response messages, confidentiality is assured only against an adversary external to the group. However, as discussed in [17], both limitations are practically acceptable in many application scenarios, since members of a multicast group are reasonably assumed to be trusted, and are not prone to tamper with messages from other group members. Furthermore, in many LLNS use cases, group members even belong to a common authority and are configured by a trusted commissioner.

In the presence of applications that inevitably require data source authentication, or assume that group members are not supposed to trust one another, application layer solutions such as digital signatures can be explicitly adopted. However, digital signatures are well known to be quite honerous from a computation standpoint, not to mention that size of signed messages increases due to the signature appending [3]. For instance, RSA-1024 requires the presence of 128 additional bytes per message, so resulting in a considerable communication overhead. Performance of digital signatures can be ameliorated by relying on Elliptic Curve Criptography (ECC) [6]. For instance, ECC-160 is roughly an order of magnitude faster than RSA, and results in 40 additional bytes per message, although providing the same level of security. Of course, as a final alternative approach, unicast DTLS sessions can still be adopted to provide source authenticity by protecting group responses altogether.

\section{KEY MANAGEMENT}

As stated in [17], DTLS-based multicast communication should rely on a secure mechanism aimed at distributing keying material, multicast security policies, and security parameters to the multicast group. Also, the $G C$ is indicated as primarily responsible for pro- 
viding the GSA to group members. However, the actual establishment of a GSA is not addressed in [17], and will be part of a future IETF activity dedicated to the design of a generic key management scheme, preferably based on requirements and recommendations defined in [9][13][19]. In this section, we propose a possible key management policy to renew the group security material and provide the current GSA to new group members upon their joining. In particular, we refer to the IETF multicast scheme described in [17] and the extension we have presented in Section 4.

We recall that the actual group key material is computed from the SecurityParameters derived from the GSA, with particular reference to the premaster secret, the client random value and the server random value. Hence, group key renewal can be easily performed by securely providing the group members with a new premaster secret. After that, the latter can be used to renew the group key material, while all other settings and information in the GSA, e.g. the adopted cryptosuite, can remain unchanged. In the following, we consider the $G C$ to be an additional member of the multicast group, and to be configured as a sender node.

Periodical rekeying. Renewing the group key material in a periodical fashion is recommended in order to discourage an external adversary from performing exhaustive key search or traffic analysis. This assumes that the group has not been compromised, i.e. a possible adversary has not taken any group member under her control. The amount of time between two consecutive occurrences of periodical rekeying should be properly defined considering the application requirements, and the perceived level of threat in the group. Of course, the more frequent the periodical rekeying, the less the damage due to possibly compromised group key material.

In order to perform a periodical rekeying, the $G C$ can broadcast a new securely generated premaster secret to group members. This can be done by broadcasting a single DTLS multicast message, protected by means of the current server write parameters as any other multicast message transmitted to the group. Once it has been received, the new premaster secret value is stored in the GSA by all group members, and used to renew the current group key material.

Such an approach relies in turn on the commonly shared group key material, and thus, as discussed in Section 4.3, cannot assure source authenticity of rekeying messages. That is, group members cannot be sure that a rekeying message has been actually sent by the $G C$. In order to address this issue, values for the random field of new premaster secrets can be generated by the $G C$ as elements of a reversed hash chain, an authentication mechanism derived from Lamport's one-time password [12]. The advantage of such an approach is that the most recently released element in the chain can be efficiently authenticated by computing its hash, and verifying that the result is equal to the previously released element in the chain. Therefore, upon creating the multicast group, it is sufficient that the $G C$ provides all members with the head element of the hash chain in an authenticated way, e.g. off-line or through a predefined pointto-point authenticated channel. Then, all other chain elements can be automatically and efficiently authenticated by group members.

Nodes' joining. In the presence of a new node joining the group, the rekeying procedure is required to assure backward security, whose importance has been explicitly stressed in [13]. Specifically, the joining node must not be able to access any group communica- tion which took place before its joining. As a first step, currently present group members can be rekeyed according to the same periodical rekeying procedure described above. After that, the $G C$ can provide the updated GSA containing the new premaster secret value to the joining node, which can then complete the join process.

In particular, the $G C$ should provide the joining node with the $G S A$ through a secure communication channel. As a possible approach, upon contacting the Resource Directory service to gain knowledge of the $G C$ address, the joining node could retrieve a public certificate associated to the $G C$, use the retrieved public key $K_{G C}^{+}$ to establish a secure channel with the $G C$, and securely obtain the GSA. As an alternative approach, the joining node can establish a DTLS session with the $G C$, and receive the $G S A$ as a sequence of protected DTLS records.

Nodes' leaving. A group member can decide to leave the multicast group, for instance when its mission is concluded or its membership is expired. Also, it can be forced to leave, in case it has been found to be compromised or it is suspected so. Then, remaining group members must be securely provided with updated group key material. This is vital in order to assure forward security, whose importance has been explicitly stressed in [13]. Specifically, the leaving node must not be able to access group communication which takes place after its departure from the group. However, the most important and difficult aspect of such a rekeying process is to prevent the leaving node from taking part in the rekeying process itself, i.e. from accessing the new security material during its distribution. In particular, since the leaving node is aware of the current group key material, the latter can not be used to securely distribute a new premaster secret to the remaining nodes.

Security considerations in [17] state that the $G C$ is assumed to share a different pairwise symmetric key with every member of the group. Although more details are not provided, it is reasonable to assume that such a key is established between the two entities during the join process. Then, upon a node's leaving, the $G C$ could rely on such pairwise keys to distribute a new premaster secret to all remaining nodes, in a one to one fashion. However, such a unicast approach lacks of efficiency, since it requires a number of rekeying messages which linearly grows with the number of nodes in the group, hence not scaling well with the group size. As an alternative, it is possible to rely on application level schemes for group rekeying, which result to be more efficient and display high scalability with the number of nodes in the group [5][8].

\section{CONCLUSION}

In this paper, we have considered the DTLS-based multicast communication method recently proposed by the IETF. In particular, we have highlighted that it displays inefficiencies in protecting unicast group responses to multicast messages. Then, we presented an extension to protect group responses according to the same group communication scheme and by reusing the same group security material. Our proposal does not break current standards, and does not require to establish additional unicast DTLS sessions among group members, thus preserving high communication performance within the group and limiting storage overhead on group members. Furthermore, we have described a suitable key management policy to perform provisioning and renewal of group key material. Future work will consist in implementing multicast communication support for the DTLS protocol, and experimentally evaluating performance in the presence and in the absence of our extension. 


\section{Acknowledgment}

The author would like to thank Christian Gehrmann and Ludwig Seitz for their comments and suggestions. A special thank goes to Sandeep Kumar and Akbar Rahman for the useful discussions.

\section{REFERENCES}

[1] Constrained RESTful Environments (CoRE). https://datatracker.ietf.org/wg/core/. Last accessed: 16 July 2014.

[2] DTLS In Constrained Environments (DICE). http://datatracker.ietf.org/wg/dice/. Last accessed: 16 July 2014.

[3] A. J. Menezes, P. C. van Oorschot and S. A. Vanstone. Handbook of Applied Cryptography. CRC Press, Boca Raton, FL, USA, 2001.

[4] A. Rahman and E. Dijk. Group Communication for CoAP, draft-ietf-core-groupcomm-21 (Work in progress). Internet Engineering Task Force, July 2014.

[5] C. K. Wong, M. Gouda and S. S. Lam. Secure Group Communications Using Key Graphs. IEEE/ACM Transactions on Networking, 8(1):16-30, February 2000.

[6] Certicom Research. Standards for Efficient Cryptography SEC 1: Elliptic Curve Cryptography. Certicom Research, May 2009.

[7] E. Rescorla and N. Modadugu. RFC 6347, Datagram Transport Layer Security Version 1.2. Internet Engineering Task Force, January 2012.

[8] G. Dini and M. Tiloca. HISS: A HIghly Scalable Scheme for Group Rekeying. The Computer Journal, 56(4):508-525, April 2013.

[9] H. Harney, A. Colegrove and G. Gross. RFC 4535, GSAKMP: Group Secure Association Key Management Protocol. Internet Engineering Task Force, June 2006.

[10] J. Postel. RFC 768, User Datagram Protocol. Internet Engineering Task Force, August 1980.

[11] J. Salowey, A. Choudhury and D. McGrew. RFC 5288, AES Galois Counter Mode (GCM) Cipher Suites for TLS. Internet Engineering Task Force, August 2008.

[12] L. Lamport. Password Authentication with Insecure Communication. Commununications of the ACM, 24(11):770-772, November 1981.

[13] M. Baugher, R. Canetti, L. Dondeti and F. Lindholm. RFC 4046, Multicast Security (MSEC) Group Key Management Architecture. Internet Engineering Task Force, April 2005.

[14] O. Garcia-Morchon, S. L. Keoh, S. Kumar, P. Moreno-Sanchez, F. Vidal-Meca and J.H. Ziegeldorf. Securing the IP-based Internet of Things with HIP and DTLS. In Sixth ACM Conference on Security and Privacy in Wireless and Mobile Networks, WiSec '13, pages 119-124, New York, NY, USA, 2013. ACM.

[15] R. Hummen, H. Wirtz, J.H. Ziegeldorf, J. Hiller and K. Wehrle. Tailoring end-to-end IP security protocols to the Internet of Things. In 21st IEEE International Conference on Network Protocols (ICNP), pages 1-10, October 2013.

[16] S. Cheshire and M. Krochmal. RFC 6763, DNS-Based Service Discovery. Internet Engineering Task Force, February 2013.

[17] S. Keoh, S. Kumar, E. Dijk and A. Rahman. DTLS-based Multicast Security for Low-Power and Lossy Networks (LLNs), draft-keoh-dice-multicast-security-08 (Work in progress). Internet Engineering Task Force, July 2014.
[18] T. Dierks and E. Rescorla. RFC 5246, The Transport Layer Security (TLS) Protocol Version 1.2. Internet Engineering Task Force, August 2008.

[19] T. Hardjono and B. Weis. RFC 3740, The Multicast Group Security Architecture. Internet Engineering Task Force, March 2004.

[20] T. Kothmayr, C. Schmitt, W. Hu, M. Brünig and G. Carle. DTLS based security and two-way authentication for the Internet of Things. Ad Hoc Networks, 11(8):2710-2723, 2013.

[21] Z. Shelby, C. Bormann and S. Krco. CoRE Resource Directory draft-ietf-core-resource-directory-01 (Work in progress). Internet Engineering Task Force, December 2013.

[22] Z. Shelby, K. Hartke and C. Bormann. RFC 7252, Constrained Application Protocol (CoAP). Internet Engineering Task Force, June 2014. 